\title{
Microwave Processing in Diagnostic Electron Microscopy
}

\author{
Ross G. Gerrity and George W. Forbes \\ Department of Pathology, BF 152, Medical College of Georgia, Augusta, Georgia 30912
}

Transmission electron microscopy (TEM) continues to play an important role in diagnostic surgical pathology [1,2], particularly in such areas as kidney pathology and tumor diagnosis, among others. Diagnostic TEM is subject to unique time constraints and other problems not seen in other TEM applications. The diagnostic TEM laboratory must produce high-quality electron microscopy on small samples which frequently are suboptimal in fixation and tissue quality due to the pathology involved and time factors associated with biopsy and surgery. Despite these problems, the diagnosis must be done as rapidly as possible, and rapid "turnaround" times of samples are a high priority, even in conditions of high caseload. Thus technology which reduces the long processing procedures for TEM samples could be of significant benefit in reducing turnaround time in the diagnostic TEM laboratory. We therefore compared turnaround times of pathology cases processed with traditional routine methods with those processed using a microwave oven for all tissue processing stages (fixation, dehydration, embedding, polymerization).

As shown in Table I, a total of 605 cases each were processed by microwave and routine methods, and the respective turnaround times noted. The cases in each category were processed within the same time span, and the figures have been corrected for non-working days (holidays and weekends). The average turnaround times calculated from these data were $2.5 \pm 1.1$ days for microwave samples vs. 3.5 \pm 1.4 days for routinely-processed samples. Although the difference in means was not statistically significant, $83 \%$ of microwave cases were completed in 3 days, 59\% in 2 days and $16 \%$ in 1 day, compared to $58 \%, 25 \%$ and $0 \%$, respectively, for routinely-processed cases. Additionally, less than $1 \%$ of microwave cases took $>5$ days to complete compared to $10 \%$ for routine processing.

Thus, from the practical standpoint, the microwave technique significantly reduced the maximum turnaround time and increased the number of cases completed in 2 days or less by 3 -fold. It also allowed cases to be completed in less than 1 day on a routine basis, which is not possible using routine methods. In our hands, microwaved blocks were indistinguishable from routinely-processed blocks in sectioning, staining and ultrastructural appearance. Of particular importance, ultrastructural features necessary for diagnoses were identical to those seen in routinely-processed samples (Fig. 1a-d). Blood cells and granules (Fig. 1a), plasma membranes, microvilli and cilia (Fig. 1b,c), microtubules and cytoplasmic filaments (Fig. 1d) as well as all other cellular organelles showed excellent preservation. Moreover, in cases difficult to embed, such as tumors with high lipid and collagen content or skin punch biopsies, microwave embedment was superior to that obtained by routine processing. The major drawback of microwave processing in the diagnostic TEM laboratory is that it is so rapid that cases are prepared more rapidly than they can be sectioned and examined, and a technician must be committed full-time to the microwave during processing and is thus not available for other duties, including sectioning. Thus to optimize the benefits of microwave processing, it must be scheduled according to the technical priorities of the day.

[1] J. Lloreta-Trull et al., Ultrastruct. Pathol. 24 (2000) 105-108.

[2] R. Kandel et al., Ultrastruct. Pathol. 22 (1998) 141-146. 


\begin{tabular}{l|c|c|c|c|c|c|c|c}
\hline & \multicolumn{7}{|c|}{ Number of Days } & \multicolumn{3}{c}{ Total Cases } \\
\hline No. Microwave Cases & 1 & 2 & 3 & 4 & 5 & 6 & Th \\
No. Regular Cases & 0 & 262 & 148 & 58 & 38 & 5 & 0 & 605 \\
\hline
\end{tabular}

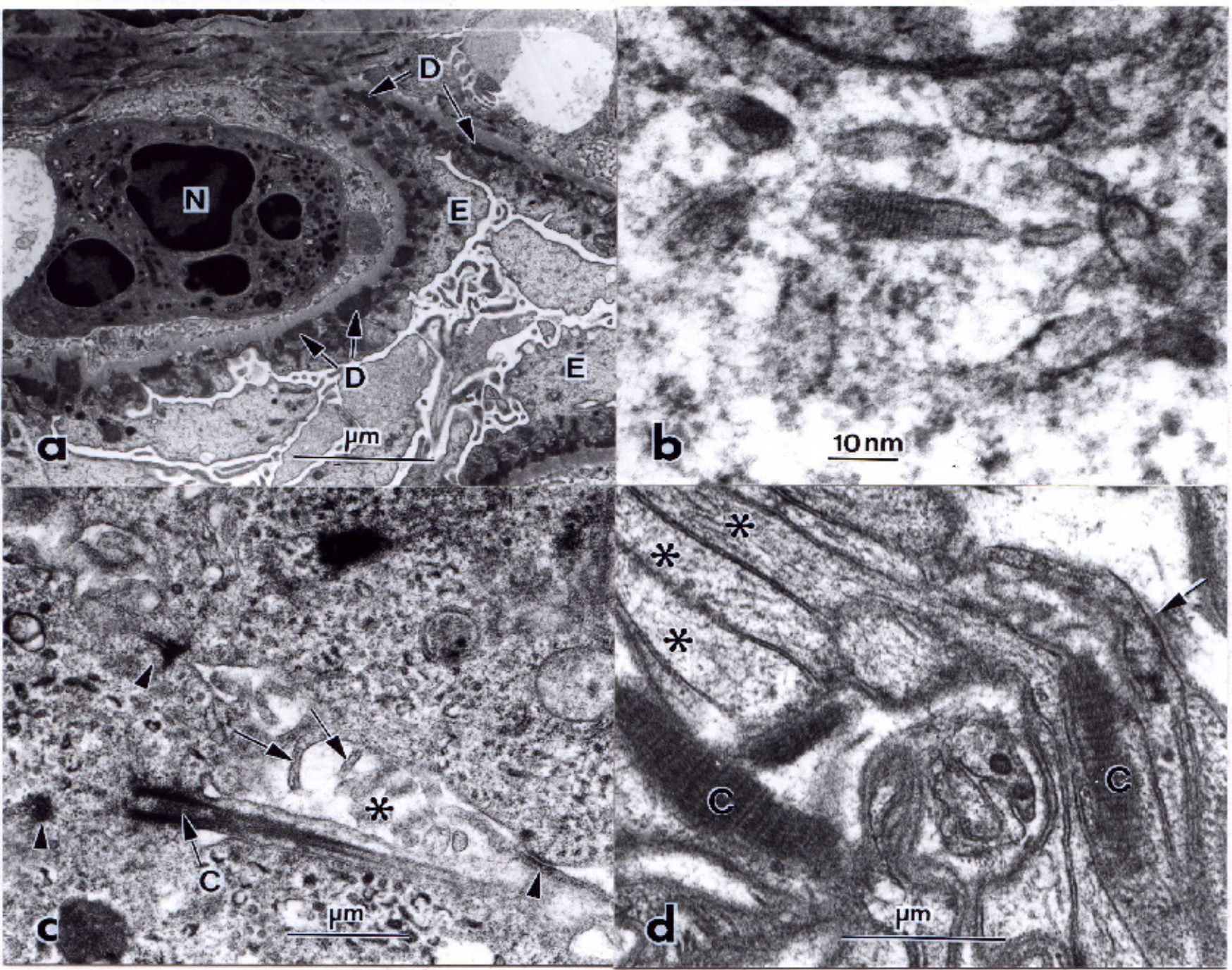

Figure 1. Ultrastructure of human biopsy specimens prepared with microwave processing from (a) renal glomerulus from lupus nephritis patient showing subepithelial dense deposits (D), effacement of foot processes in epithelial layer (E) and neutrophil (N) in capillary; (b) melanoma, showing striated structure of a stage 2 melanosome essential for diagnosis; (c) ovarian adenocarcinoma, showing microvilli (arrows) and a cilium (C) extending into acinar space $\left({ }^{*}\right)$ bounded by junctional complexes (arrowheads); (d) schwannoma, showing typical layered cell processes (*), fragmented external lamina (arrow), microtubules and cytoplasmic filaments and fibrous long spacing collagen. 